\title{
Electrochemical Behavior and Analytical Applications of Electronically Type-Sorted Carbon Nanotube Electrode
}

\author{
Yuki Inoue, Tatsuya Hoshino, and Hitoshi Muguruma* \\ Shibaura Institute of Technology, 3-7-5 Toyosu, Koto-ku, Tokyo 135-8548, Japan
}

(Received July 31, 2015; accepted September 7, 2015)

Keywords: electronically type-sorted single-walled carbon nanotube, plasma-polymerized film, electrochemical sensor, nicotinamide adenine dinucleotide

The electrochemical behavior and analytical applications of an electrode with electronically typesorted (metallic and semiconducting) single-walled carbon nanotubes (SWCNTs) were examined. The effect of the electronic type of SWCNTs on the electrochemical behavior of nicotinamide adenine dinucleotide (NADH) as a model agent was investigated. The electrode configuration contained SWCNTs sandwiched between 3-5-nm-thick acetonitrile plasma-polymerized thin films. Cyclic voltammetry measurements demonstrated that the current due to the NADH oxidation of the electrode with semiconducting SWCNTs was much larger than that for electrodes with only metallic or unsorted SWCNTs. Electrochemical impedance spectroscopy measurements and atomic force microscopy observations suggested that the electrode with semiconducting SWCNTs formed a better electron transfer network than the electrodes with metallic or unsorted SWCNTs. Chronocoulometry measurements indicated that there was no distinct difference between the diffusion coefficients of NADH with the metallic and semiconducting SWCNT electrodes. Therefore, it was concluded that the semiconducting SWCNT electrode is more suitable than the metallic and unsorted SWCNT electrodes for electrochemical oxidation in terms of catalysis and electron transport. The semiconducting SWCNT electrode exhibited excellent performance for the sensing application of NADH detection with a significantly wide linear range of 59-5800 $\mu \mathrm{M}$ compared with that for conventional NADH sensors. Other significant performance characteristics are a sensitivity of $176 \mu \mathrm{A} \mathrm{mM}^{-1} \mathrm{~cm}^{-2}$ (defined as the slope of the current vs concentration plot at $+0.4 \mathrm{~V}$ ), a detection limit of $1.1 \mu \mathrm{M}$ [signal-to-noise ratio $(S / N)=3$ ], and a response time of $6 \mathrm{~s}$.

\section{Introduction}

Single-walled carbon nanotubes (SWCNTs) are the result of folding graphene layers into carbon cylinders. They consist of hollow cylindrical graphene layers with a geometrically unique structure and display excellent electrical conductivity, excellent catalytic activity, high mechanical strength, and high chemical stability. The directional variety of the folding (chirality) results in electrically heterogeneous properties, i.e., metallic (m-) and semiconducting (s-) SWCNTs. ${ }^{(1,2)}$ The as-grown (unsorted) SWCNTs are typically a mixture of approximately onethird m-SWCNTs and two-thirds s-SWCNTs. A method for sorting SWCNTs according to their electrical properties has been developed and electronically homogeneous SWCNTs have been

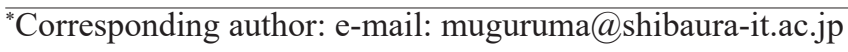


successfully harvested.(3) Consequently, there have been many reports on the applications of electronically homogeneous SWCNTs. For example, thin-film field-effect transistors (TFTs) with s-SWCNTs have been reported to have higher drive currents and larger on/off current ratios than their m-SWCNT and unsorted SWCNT counterparts. ${ }^{(3-5)}$ Chemical sensors based on s-SWCNT TFTs exhibit higher performance than those with unsorted SWCNTs. ${ }^{(6,7)}$ The sensing mechanism of those chemical and gas sensors exploits the potential change due to the electrostatic interaction between the SWCNTs and the target molecule, resulting in a change in output current. This type of sensor is categorized as a potentiometric sensor. ${ }^{(8)}$ The higher performance of s-SWCNTs than of $\mathrm{m}$-SWCNTs in TFT-based electronic devices and sensors is due to the presence of $\mathrm{m}$-SWCNTs that reduces the on-off ratio owing to the charge transport being dominated by metallic pathways.

Beyond their application as transistors, $\mathrm{m}$ - and s-SWCNTs also differ in many other properties, such as chemical reactivity and the adsorption of molecules. ${ }^{(1)}$ The sensing mechanism of chemical sensors with such SWCNTs is the detection of current change due to molecular oxidation, and such sensors are categorized as amperometric sensors. ${ }^{(8)}$ However, there have been only a few studies on amperometric sensors (catalytic oxidation for target molecules) with electronically typesorted SWCNTs. We previously demonstrated an amperometric enzyme biosensor; ${ }^{(9)}$ however, the main focus was the combination of two catalysts of SWCNTs and an enzyme. We envision that a fundamental understanding of the electrochemical behavior and analytical applications of electronically type-sorted SWCNT will be achieved through further investigations.

We have focused on the electrochemical detection of the reduced form of $\beta$-nicotinamide adenine dinucleotide (NADH) because the $\mathrm{NAD}^{+} / \mathrm{NADH}$ couple is the cofactor system for a large number of dehydrogenase enzymes and a component of biomarker systems. Recently, there has been an increase in the number of reports of NADH sensing with SWCNTs because of their excellent electron transfer properties and catalytic activities. ${ }^{(10-18)}$ In this article, the effect of electronically type-sorted SWCNTs on electrochemical performance is addressed and their analytical applications are examined. The investigation of the effectiveness of electrochemical sensors with electronically type-sorted SWCNTs is a challenging task because these chemical sensors work in aqueous media, unlike other electronic ${ }^{(3-5)}$ and electrochemical ${ }^{(7)}$ devices that work in the atmosphere. Here, we adopt a simple layer-by-layer process based on a plasma-polymerized film (PPF). ${ }^{19,20)}$ The PPF and plasma modifications act as an inactive matrix to integrate the SWCNT electrode and provide a well-defined nanostructure and a versatile platform for catalytic and analytical applications.

\section{Materials and Methods}

\subsection{Materials}

Electronically type-sorted SWCNTs (IsoNanotube-M and IsoNanotube-S) were purchased from Nanointegris Inc. (Skokie, IL, USA). ${ }^{(3)}$ Solutions of unsorted SWCNTs for a control experiment (PureTubes) were also purchased from Nanointegris Inc. (Skokie, IL, USA). The solutions included a surfactant composed of a mixture (1:3) of sodium dodecyl sulfate and $1 \%(\mathrm{w} / \mathrm{v})$ sodium cholate. The as-purchased solutions of sorted and unsorted SWCNTs were concentrated to achieve the desired concentrations. Acetonitrile, ammonia, potassium dihydrogenphosphate, disodium hydrogenphosphate, D-glucose, hydrogen peroxide, and ascorbic acid were purchased from Kanto Chemical Co., Inc. (Tokyo, Japan). NAD-dependent glucose dehydrogenase (GDH) from Bacillus $s p$. (EC 1.1.1.47) and NADH (reduced form) were purchased from Wako (Osaka, Japan). NAD ${ }^{+}$ (oxidized form) was purchased from Sigma (St. Louis, MO, USA). 


\subsection{Fabrication procedure}

The electrochemical device was formed on a 0.15-mm-thick glass substrate. All metal layers were sputtered with a plasma generator (VEP-1000, Ulvac, Inc., Tokyo, Japan). A 40-nm-thick chromium intermediate layer was used to promote the adhesion of the gold layer. The dimensions of the opening for the working electrode were $5 \times 5 \mathrm{~mm}^{2}$.

The plasma generator was used to deposit a 2-nm-thick acetonitrile PPF layer (first PPF layer) onto the sputtered $\mathrm{Au}$ electrode at a power of $150 \mathrm{~W}$ and a pressure of $0.6 \mathrm{~Pa}$. Aqueous solutions of the sorted SWCNTs containing 1\% surfactant were used. The SWCNT solution $\left(1 \mathrm{mg} \mathrm{mL}^{-1}\right.$, optimized) was dropped onto the PPF surface and dried in a vacuum oven. The thickness of the resultant sorted-SWCNT film was 50-70 nm. Finally, the enzyme-adsorbed surface was overcoated with a 6-nm-thick acetonitrile PPF layer (second layer). The deposition parameters were as follows: power $150 \mathrm{~W}$; pressure $0.6 \mathrm{~Pa}$; exposure time $150 \mathrm{~s}$. The immobilization procedure for enzyme GDH is described in the literature. ${ }^{(9)}$

\subsection{Measurements}

Electrochemical measurements were performed with an electrochemical analyzer (ALS Instruments, 701A West Lafayette, IN, USA) using a three-electrode configuration. Reference ( $\mathrm{Ag} / \mathrm{AgCl}, \mathrm{RE}-1 \mathrm{C})$ and counter (platinum wire) electrodes were purchased from Bioanalytical Systems Inc. Electrochemical measurements were conducted in a $5 \mathrm{~mL}$ vessel at room temperature $\left(20{ }^{\circ} \mathrm{C}\right)$ using a phosphate buffer $(20 \mathrm{mM}, \mathrm{pH} 7.4)$ as the supporting electrolyte, to which stock NADH solutions of $0.3,3$, or $30 \mathrm{mM}$ were successively added to prepare samples with designated concentrations. Electrochemical measurements were performed at least four times. Electrochemical impedance spectroscopy was conducted in the frequency range of $100 \mathrm{mHz}$ to $100 \mathrm{kHz}$ at a DC potential of $0.25 \mathrm{~V}$ and an AC perturbation of $5 \mathrm{mV}$. The electrolyte was $\mathrm{pH}$ 7.4, $20 \mathrm{mM}$ phosphate buffer solution containing $10 \mathrm{mM} \mathrm{K}_{3}\left[\mathrm{Fe}\left(\mathrm{CN}_{6}\right)\right] / \mathrm{K}_{4}\left[\mathrm{Fe}(\mathrm{CN})_{6}\right](1: 1, \mathrm{v} / \mathrm{v})$. Chronocoulometric measurements were also conducted to evaluate the diffusion coefficient of each analyte passing through the film. A potential step between 0 and $+0.5 \mathrm{~V}$ (vs $\mathrm{Ag} / \mathrm{AgCl}$ ) was applied to the working electrode at a sampling interval of $10 \mathrm{~ms}$. Atomic force microscopy (AFM) was conducted in the tapping mode using a commercial AFM system (NanoScope IIIaAFM Dimension 3000 stage system, Nihon Veeco K.K., Tokyo, Japan). The scanning tip was equipped with an ACmode super sharp chip (Nanosensors Inc., Neuchatel, Switzerland). A scanning rate of $0.6 \mathrm{~Hz}$ was employed.

\section{Results and Discussion}

\subsection{Morphological study}

The electrodes based on type-sorted SWCNTs and PPFs have a layer-by-layer structure of PPF/ SWCNT/PPF/Au. The first PPF layer on the Au electrode acts as a scaffold for the formation of the SWCNT layer; therefore, a SWCNT layer cannot be obtained without the first PPF layer. ${ }^{(19)}$ The method used for the immobilization of the SWCNTs on the electrode surface is to place a droplet of SWCNT solution onto the electrode surface and allow it to evaporate. The morphological features of the SWCNT layer (SWCNT/PPF/Au) surfaces were observed by AFM, as shown 
in Fig. 1. There were few differences in network morphology between the sorted and unsorted SWCNT surfaces. This is in contrast to the report by Wang et al. ${ }^{(21)}$ where larger bundles were observed in the unsorted SWCNT surface than in the sorted SWCNT surface. This is attributed to the presence of the surfactant, which acts to unbundle the SWCNTs. The surface roughness of the unsorted SWCNT layer was greater than that of the sorted SWCNT layer, which suggests that the unsorted SWCNT layer may exhibit geometrically structural heterogeneity. Thus, the sorting process for density gradient ultracentrifugation not only provides electronic homogeneity but also structural homogeneity. There were no significant differences in alignment or uniformity between m-SWCNTs and s-SWCNTs, although the s-SWCNT network was slightly denser than the m-SWCNT network. This is related to the higher oxidation current exhibited by the s-SWCNT electrode than by the m-SWCNT and unsorted SWCNT electrodes, as discussed in the following section. Finally, the second PPF was directly deposited onto the SWCNT layer (PPF/SWCNT/PPF/ $\mathrm{Au}$ ). The second PPF is used to change the electrode from hydrophobict to hydrophilic, while not significantly altering the electrical properties. ${ }^{(19)}$

\subsection{Electrochemical behavior}

\subsubsection{Cyclic voltammetry (CV)}

$\mathrm{CV}$ is a useful tool for the fundamental evaluation of SWCNT electrodes. CV was used to investigate the effect of the electronic type of the SWCNT layer on the electrochemical response of the SWCNT electrode toward NADH oxidation. Firstly, the effect of the surfactant, which is mandatory for the sorting process, ${ }^{(3)}$ was investigated. Figures 2(a) and 2(a') show CV profiles for the unsorted SWCNT electrodes with and without the surfactant. The current due to the NADH oxidation of the SWCNT electrode with surfactant was 10-fold larger than in the case without surfactant, which suggests that the surfactant is responsible for boosting the electrochemical response. The surfactant facilitates the unbundling of the SWCNTs and contributes to the

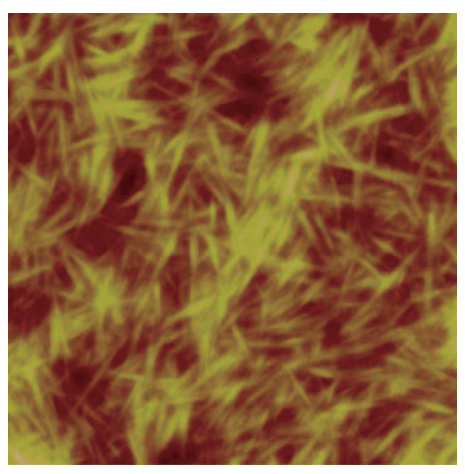

(a)

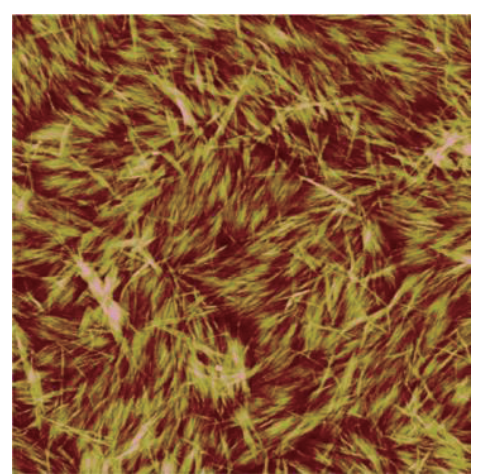

(b)

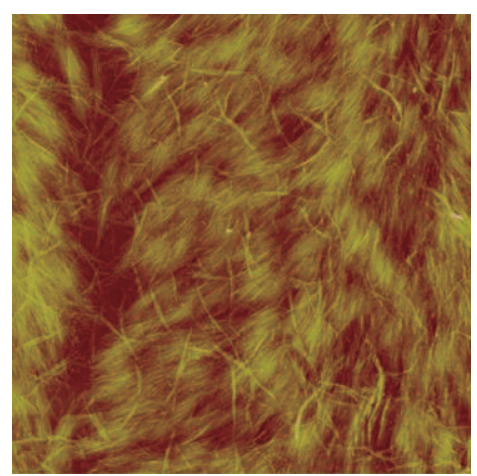

(c)

Fig. 1. (Color online) AFM images of the surfaces of (a) unsorted SWCNT, (b) m-SWCNT, and (c) s-SWCNT layers formed by drop-casting the SCWNT solution $(1 \mathrm{mg} / \mathrm{mL})$. Horizontal scale: $10 \times 10 \mathrm{~mm}^{2}$. Vertical scale: (a) 150, (b) 30, and (c) $30 \mathrm{~nm}$. 

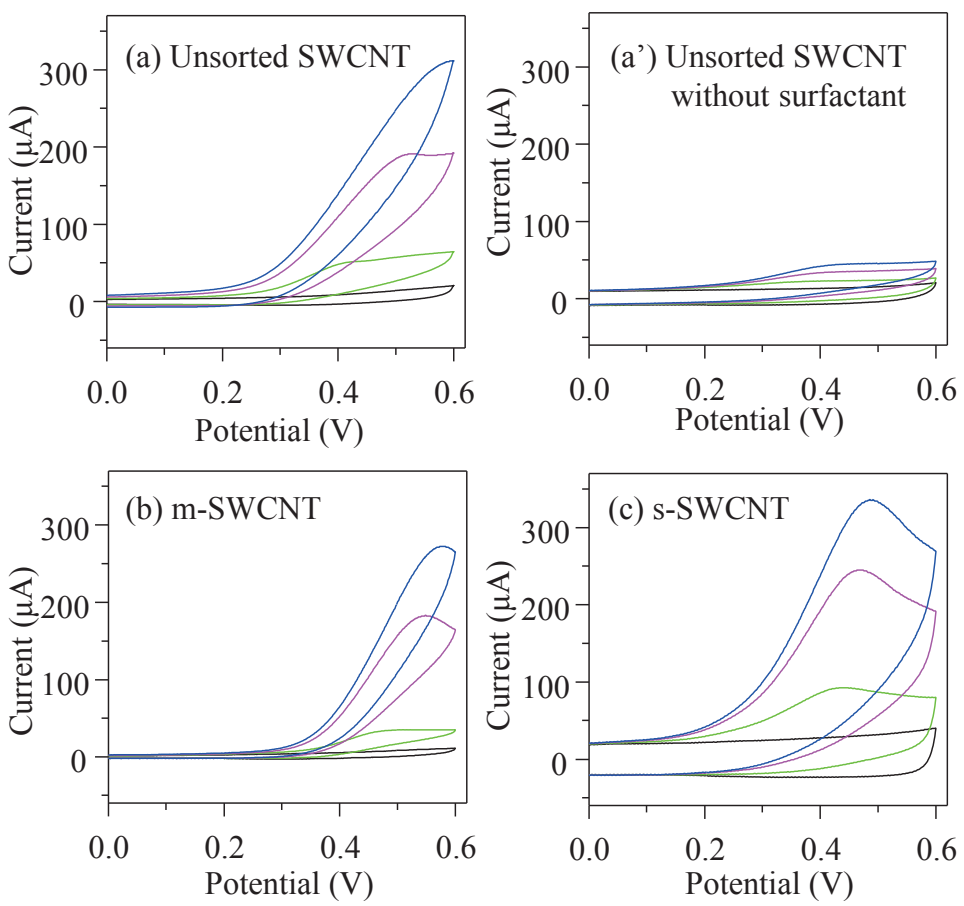

Fig. 2. (Color online) CV profiles for (a) unsorted SWCNT, (a') unsorted SWCNT without surfactant, (b) $\mathrm{m}-\mathrm{SWCNT}$, and (c) s-SWCNT electrodes in the absence and presence of NADH. The sweep rate was $50 \mathrm{mV} \mathrm{s}^{-1}$ and the NADH concentrations were $0,1.7,4.2$, and $5.8 \mathrm{mM}$. The electrolyte used was $\mathrm{pH} 7.4,20 \mathrm{mM}$ phosphate buffer solution. The scan rate was $50 \mathrm{mV} \mathrm{s}^{-1}$. The surface area of the electrode was $25 \mathrm{~mm}^{2}$.

homogeneous morphology, which reduces the barrier for NADH access and oxidation. No negative factors from the surfactant, such as the hydrophobic naphthenic groups of the sodium cholate surfactant blocking the electronic signal, ${ }^{(22)}$ were observed.

Figure 2 shows CV profiles for the electrodes with unsorted SWCNTs, m-SWCNTs, and s-SWCNTs. In all electrodes, the increase in current due to the oxidation of NADH was observed at around $+0.45 \mathrm{~V}$. NADH oxidation on conventional electrodes requires a positive potential as high as $1 \mathrm{~V}$; (23) therefore, SWCNTs reduce the activation energy of the electrochemical reaction on the surfaces. The electrons generated are transported from the SWCNTs to the Au electrode, which results in an increase in current. This polarized potential is similar to those of other electrodes with CNTs and edge-plane pyrolytic graphite. ${ }^{(24)}$ Therefore, this effect is due to the presence of not only the electronically type-sorted SWCNTs but also the CNTs. The mechanism for electrochemical $\mathrm{NADH}$ oxidation in the proposed system is as follows:

$$
\begin{aligned}
& \text { SWCNTs }+\mathrm{NADH} \rightarrow \text { SWCNTs } \cdot \mathrm{NADH}, \\
& \text { SWCNTs } \cdot \mathrm{NADH} \rightarrow \mathrm{SWCNTs} \cdot \mathrm{NADH}^{\bullet}{ }^{+}+\mathrm{e}^{-}, \\
& \text {SWCNTs } \cdot \mathrm{NADH}^{\bullet}{ }^{+} \rightarrow \mathrm{SWCNTs} \cdot \mathrm{NAD} \bullet+\mathrm{H}^{+},
\end{aligned}
$$




$$
\mathrm{SWCNTs} \cdot \mathrm{NAD} \bullet \rightarrow \text { SWCNTs} \cdot \mathrm{NAD}^{+}+\mathrm{e}^{-},
$$

where NADH diffuses and adsorbs onto the electrode surface and is then electrochemically oxidized by an electron transfer-chemical reaction-electron transfer (ECE) mechanism. ${ }^{(25,26)}$ According to this ECE mechanism, the first step of the NADH electrochemical oxidation is an irreversible heterogeneous electron transfer. This is supported by the sigmoidal CV without a reduction peak (see Fig. 2). In this step, one electron is lost and a cation radical, $\mathrm{NADH}^{\bullet^{+}}$, is produced, as in Eq. (2). The neutral radical NAD• is produced through a first-order deprotonation reaction of $\mathrm{NADH}^{\bullet}{ }^{+}$in Eq. (3). A continuous reaction for electron transfer to form NAD• is induced by the second heterogeous electron transfer in Eq. (4). In this system, SWCNTs serve as an electrocatalyst for NADH oxidation and an electron conductor. To summarize Eqs. (1)-(4), SWCNTs catalyze the oxidation of NADH to NAD+ (oxidized form of NADH), and the Au electrode detects two electrons, as follows:

$$
\mathrm{NADH} \stackrel{\text { SWCNTs }}{\rightarrow} \mathrm{NAD}^{+}+\mathrm{H}^{+}+\mathrm{e}^{-} .
$$

When comparing SWCNT electrodes by electronic type, the increase in current due to the NADH oxidation of various SWCNT electrodes is in the following order: s-SWCNT $>$ unsorted SWCNT $>$ m-SWCNT. The s-SWCNT electrode did not only exhibit the best response but also showed the most distinct oxidation peak at around $+0.45 \mathrm{~V}$. The electrochemical properties are thus considered to be dominated by s-SWCNTs.

\subsubsection{Electrochemical impedance spectroscopy (EIS)}

EIS is a highly effective method used to probe the surface features of electrodes. EIS was used to investigate the electron transfer properties of the SWCNT electrodes. Figure 3 shows typical impedance spectra, in the form of Nyquist plots, for these SWCNT electrodes. The small semicircular domain at high frequencies corresponds to an electron-transfer-limited process and the straight line at low frequencies corresponds to a diffusion process. The Randles equivalent circuit (Fig. 3, inset) was selected to fit the impedance data, where $R_{\mathrm{s}}$ is the solution resistance, $R_{\mathrm{et}}$ is the electron transfer resistance, $W$ is the Warburg impedance, and $C_{\mathrm{dl}}$ is the double-layer capacitance. The $R_{\mathrm{et}}$ of $6.5 \mathrm{k} \Omega$ obtained for the s-SWCNT electrode, which was calculated from the diameter of the semicircular domain in the Nyquist plot, was much smaller than that for the m-SWCNT electrode $\left(R_{\mathrm{et}}=14 \mathrm{k} \Omega\right)$ and was slightly smaller than that for the unsorted SWCNT electrode $\left(R_{\mathrm{et}}\right.$ $=7.0 \mathrm{k} \Omega$ ). The EIS data are not consistent with the electrical properties of the bulk SWCNTs, but are consistent with those of a network of SWCNTs. Thus, although bulk m-SWCNTs have a higher Fermi level density than bulk s-SWCNTs, electron transport to the sensing electrode through the hopping process is more easily achieved in the s-SWCNT network than in the m-SWCNT network. The similarity of the $R_{\mathrm{et}}$ values of the s-SWCNT and unsorted SWCNT electrodes is probably due to the electron transfer properties being dominated by the s-SWCNT network, where the unsorted SWCNTs consist of m-SWCNT:s-SWCNT $=1: 2$. 


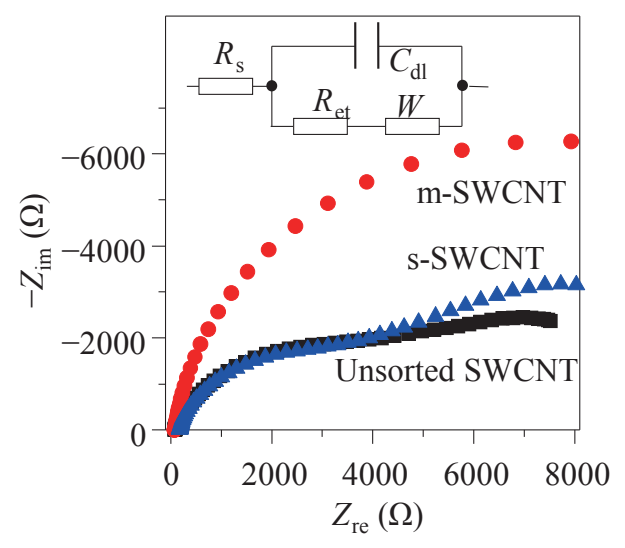

Fig. 3. (Color online) Nyquist plots and equivalent electrical circuit (inset) for unsorted SWCNT, m-SWNT, and s-SWCNT electrodes. The electrolyte was $\mathrm{pH} 7.4,20 \mathrm{mM}$ phosphate buffer solution containing $10 \mathrm{mM} \mathrm{K}_{3}\left[\mathrm{Fe}\left(\mathrm{CN}_{6}\right)\right] / \mathrm{K}_{4}\left[\mathrm{Fe}(\mathrm{CN})_{6}\right](1: 1)$.

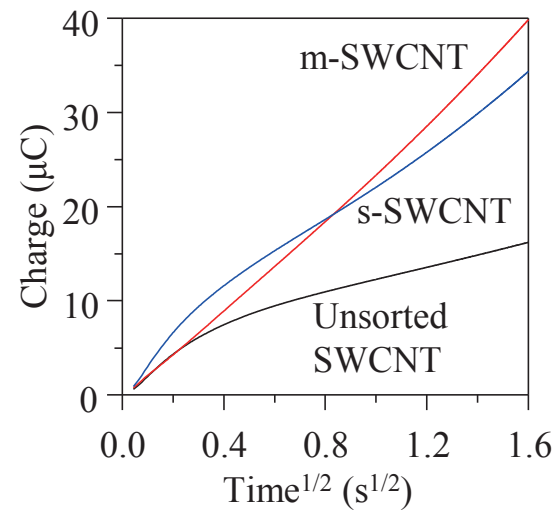

Fig. 4. (Color online) Chronocoulometric plots of NADH $(0.3 \mathrm{mM})$ at unsorted SWCNT, m-SWCNT, and s-SWCNT electrodes. The potential step was from 0 to $+0.5 \mathrm{~V}$ and the sampling time was $10 \mathrm{~ms}$.

\subsubsection{Chronocoulometry}

Chronocoulometric measurements were also conducted to evaluate the diffusion coefficient of each analyte passing through the sensing layer. A potential step between 0 and $+0.5 \mathrm{~V}$ ( $\mathrm{vs} \mathrm{Ag} / \mathrm{AgCl}$ ) was applied to the working electrode at a sampling interval of $10 \mathrm{~ms}$. The diffusion coefficient $\mathrm{D}$ was determined by integrating the Cottrell equation:(27)

$$
Q=n F A \Gamma_{0}+Q_{\mathrm{dl}}+2 n F A C D^{1 / 2} t^{1 / 2} \pi^{-1 / 2},
$$

where $n$ is the number of electrons transferred to the electrode, $F$ is the Faraday constant, $A$ is the area of the electrode, $C$ is the concentration of the analyte, $t$ is the time after the potential step, $\Gamma_{0}$ is the surface concentration of the analyte, and $Q_{\mathrm{dl}}$ is the double-layer charge.

Chronocoulometry was conducted to determine the apparent diffusion coefficient $\left(D_{\text {app }}\right)$ of a species. $D_{\text {app }}$ determined by this procedure incorporates (i) the diffusion of the analyte from the bulk phase to the functional (SWCNT) surface, (ii) the partition between the solution and the functional surface, and (iii) the diffusion through the surface of the electrode. The charging and reaction on the surface of the electrode are so fast that they can be ignored. The chronocoulometric charge as a function of the square root of time for the oxidation of NADH at the SWCNT electrode is shown in Fig. 4. From the Anson plot, the square of the slope is proportional to $D_{\text {app }}$ [see Eq. (6)]. In this case, the slope beyond $0.4 \mathrm{~s}^{1 / 2}$ along the $x$-axis is constant (Fig. 3) and corresponds to the thickness of the PPF/SWCNT/PPF layer, which suggests that the linearity observed during the longer time period represents the diffusion process. The CV of the electrode was also investigated with a variable scan rate to determine the diffusion process. The anodic peak current and peak potential shifted in the positive direction with an increase in scan rate. The linearity of the peak current vs the square root of the potential scan rate confirmed the electrochemical irreversibility 
of the electrocatalytic reaction and indicates that this electrocatalytic process is controlled by NADH diffusion from the solution to the surface of the SWCNT layer. The diffusion coefficient for NADH through the unsorted SWCNT layer $\left(1.7 \times 10^{-7} \mathrm{~cm}^{2} \mathrm{~s}^{-1}\right)$ was smaller than those through the sorted SWCNT layers (m- and s-SWCNTs were $2.2 \times 10^{-6}$ and $1.1 \times 10^{-7} \mathrm{~cm}^{2} \mathrm{~s}^{-1}$, respectively). This is because the roughness and thickness of the unsorted SWCNT layer were larger than those of the sorted SWCNT layers (see Fig. 1). Although the diffusion coefficient of NADH through the m-SWCNT layer is similar to that through the s-SWCNT layer, the oxidation current of the s-SWCNT electrode is larger than that of the m-SWCNT electrode. This suggests that the current response is mainly dominated by electron transfer due to oxidation.

The intercept of the ordinate in Fig. 4 corresponds to the surface charge [Eq. (6)] and suggests that the difference in surface properties between the s- and m-SWCNT electrodes affects the electrochemical behavior. For the s-SWCNT electrode, the positive inset indicates a positively charged surface. ${ }^{(27)}$ This is due to the modification of the acetonitrile PPF after SWCNT layer formation. The PPFs synthesized from acetonitrile consisted of a nitrogen-rich surface containing primary and secondary amine groups. ${ }^{(20)}$ Assuming that $Q_{\mathrm{dl}}$ is zero, $\Gamma_{0}$ for the s-SWCNT layer was estimated to be $2.1 \times 10^{-10} \mathrm{~mol} \mathrm{~cm}{ }^{-2}$, which is similar to that for the unsorted SWCNT layer $(2.3$ $\times 10^{-10} \mathrm{~mol} \mathrm{~cm}^{-2}$ ) and that reported for a quinone-functional electrode. ${ }^{(24,28)}$ In contrast, $\Gamma_{0}$ for the $\mathrm{m}-\mathrm{SWCNT}$ electrode was near zero, which suggests that $C_{\mathrm{dl}}$ for the m-SWCNT electrode is smaller than those for the unsorted and s-SWCNT electrodes (see the ordinate in Fig. 3).

\subsubsection{Summary of electrochemical behavior of electronically type-sorted SWCNT electrodes}

$\mathrm{CV}$ measurements demonstrated that the current due to the NADH oxidation of the electrode with s-SWCNTs was much larger than those for the electrodes with m-SWCNTs and unsorted SWCNTs. EIS measurements and AFM observations suggested that the s-SWCNT electrode formed a better electron transfer network than those with m-SWCNTs and unsorted SWCNTs. Chronocoulometry measurements suggested that the surface kinetics for NADH oxidation is dominated by the diffusion of NADH and there is no distinct difference in the diffusion coefficient for NADH between the m-SWCNT and s-SWCNT electrodes. Therefore, we conclude that s-SWCNTs are more suitable than m-SWCNTs and unsorted SWCNTs with respect to catalysis and electron transfer for electrochemical oxidation.

\subsection{Analytical applications}

\subsubsection{NADH sensing}

A typical application of such electrochemical behavior is electrochemical sensing. NADH is an electroactive and physiological material, the sensing mechanism of which can be used according to the electrochemical oxidation shown by Eq. (5). To date, much research with SWCNT-based NADH sensors has been reported. There are mainly two approaches to the strategies of SWCNTbased NADH sensors. One is the use of an electron transfer mediator. ${ }^{(10,12,15,16,29)}$ The synergetic effect of SWCNTs and an electron transfer mediator can be used to realize a high-performance sensor. Although the use of such a mediator seems promising, the mediator still causes problems, such as leakage from the electrode surface, lack of long-term stability, and toxicity, which negatively affect its analytical applications. Therefore, the use of mediatorless sensors has also been reported as the second approach. ${ }^{(11,17,30)}$ The main issues with this approach are the use of 
a functional (inactive) matrix, such as hyaluronic acid, ${ }^{(11)} \mathrm{PPF},{ }^{(30)}$ or polyaniline. ${ }^{(17)}$ There have been other attempts to use carbon nanotubes that have been subjected to boron doping, ${ }^{(31)}$ nitrogen doping, ${ }^{(32)}$ boiling in water, ${ }^{(24)}$ and acid microwave treatment; ${ }^{(24)}$ however, these approaches are only available for multiwalled carbon nanotubes. Therefore, the proposed approach of using different electronic forms of SWCNTs is novel.

Figure 5(a) presents the amperometric responses of sensors with the sorted and unsorted SWCNT electrodes at a fixed potential of $+0.4 \mathrm{~V}$ vs Ag/ $\mathrm{AgCl}$ during NADH addition. A sequential stepwise increase in current with NADH addition was observed. The response of the s-SWCNT electrode was twice those of the m-SWCNT and unsorted SWCNT electrodes. This increase in current with a fixed potential correlated with the CV data shown in Fig. 2. Figure 5(b) shows the current as a function of the NADH concentration based on the data in Fig. 5(a). The background current (approximately $0.32 \mu \mathrm{A}$ ) is small compared with the NADH response, which indicates that there is no need to perform a baseline calibration for NADH measurement. The most significant characteristic is a very wide linear range $(59-5800 \mu \mathrm{M}, r=0.998)$ compared with the other NADH sensors based on SWCNTs, as shown in Table 1 . $^{(10-12,15-17,21,30,31)}$ At high concentrations $(>3 \mathrm{mM})$, no current saturation was observed, unlike in conventional NADH sensors. This is attributed to the effective electrochemical communication provided by the electronically homogeneous s-SWCNTs. Other significant characteristics include a sensitivity of $176 \mu \mathrm{A} \mathrm{mM}^{-1} \mathrm{~cm}^{-2}$, defined by the slope of the current vs concentration plot in the linear range (s-SWCNT electrode), a detection limit of 1.1 $\mu \mathrm{M}$ [signal-to-noise ratio $(S / N)=3$ ], and a response time of $6 \mathrm{~s}$ (to $90 \%$ of maximum response).

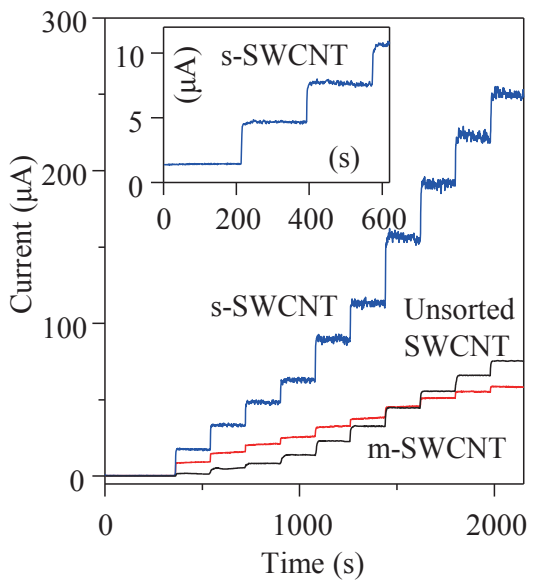

(a)

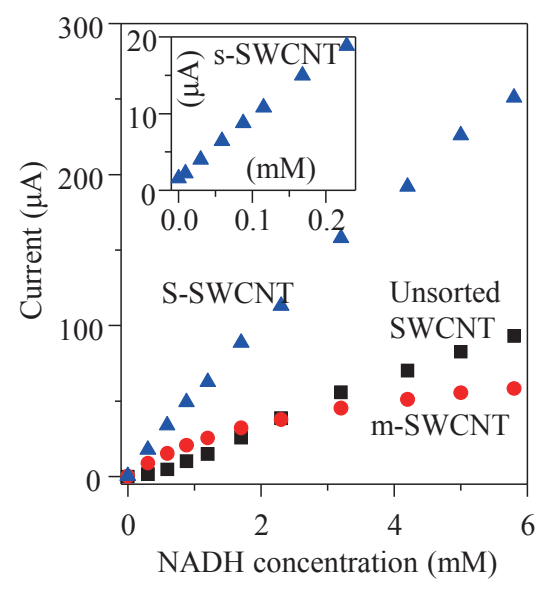

(b)

Fig. 5. (Color online) (a) Time-current response to sequential NADH addition at concentrations of 0.30, 0.59, $0.88,1.2,1.7,2.3,3.2,4.2,5.0$, and $5.8 \mathrm{mM}$ with unsorted SWCNT, m-SWCNT, and sSWNT electrodes. Inset: enlargement of region of low NADH concentration $(0.03,0.059$, and $0.088 \mathrm{mM})$ for the s-SWCNT electrode. Polarized potential: $+0.4 \mathrm{~V}$ vs $\mathrm{Ag} / \mathrm{AgCl}$. Response time: $7 \mathrm{~s}$ (to $95 \%$ of maximum level). Detection limit: $1.1 \mu \mathrm{M}$ $(S / N=3$, s-SWNT). (b) Calibration plot for NADH response using the data in (a). Inset: enlargement of the low concentration range for the s-SWCNT electrode. Correlation line in the NADH concentration range (59-5800 $\mu \mathrm{M}$, s-SWCNT); sensitivity of $176 \mu \mathrm{A} \mathrm{mM}^{-1} \mathrm{~cm}^{-2}(r=0.998, \mathrm{~s}-\mathrm{SWCNT})$. 
Table 1

Comparison of the performance parameters of NADH sensors based on SWCNTs.

\begin{tabular}{|c|c|c|c|c|c|c|c|}
\hline Electrode & $\begin{array}{c}\text { SWCNT } \\
\text {-functional- } \\
\text { ization }\end{array}$ & $\begin{array}{c}\text { Sensitivity } \\
\left(\mathrm{mAmM}^{-1}\right. \\
\left.\mathrm{cm}^{-2}\right)\end{array}$ & $\begin{array}{l}\text { Linear } \\
\text { range } \\
(\mathrm{mM})\end{array}$ & $\begin{array}{l}\text { Detection } \\
\text { limit } \\
(\mathrm{mM})\end{array}$ & $\begin{array}{c}\text { Applied } \\
\text { potential } \\
\text { (V) }\end{array}$ & $\begin{array}{c}\text { Response } \\
\text { time } \\
\text { (s) }\end{array}$ & Ref. \\
\hline \multicolumn{8}{|l|}{ Mediator-free systems } \\
\hline $\mathrm{PPF} / \mathrm{s}-\mathrm{SWCNT} / \mathrm{PPF} / \mathrm{Au}$ & Sorted & 176 & $30-5800$ & 0.9 & 0.4 & 7 & This work \\
\hline Hyaluronic acid-SWCNT/GC & Unsorted & 6.86 & N/A & 63 & 0.4 & 19 & (11) \\
\hline $\mathrm{PPF} / \mathrm{SWCNT} / \mathrm{PPF} / \mathrm{Au}$ & Unsorted & 240 & $9-2300$ & 3.9 & 0.4 & 7 & $(30)$ \\
\hline Polyaniline/SWCNT/Au ${ }^{(a)}$ & Unsorted & $\mathrm{N} / \mathrm{A}$ & $5-1000$ & 1 & 0.05 & N/A & (17) \\
\hline Boron-doped MWCNT ${ }^{(\mathrm{b})} / \mathrm{GC}$ & Boron-doped & $\mathrm{N} / \mathrm{A}$ & -1405 & 0.05 & 0.3 & 5 & $(31)$ \\
\hline $\begin{array}{l}\text { Acid-microwaved MWCNT(b)/GC } \\
\text { Mediated systems }\end{array}$ & Acid-microwaved & 12 & $10-100$ & 2.0 & 0.0 & $\sim 50$ & $(24)$ \\
\hline Poly(phenosafranin)-SWCNT/EPPG & Unsorted & 576 & $10-170$ & 0.01 & 0.0 & $<2$ & $(12)$ \\
\hline Variamine blue-SWCNT/SPE(c) & Unsorted & N/A & $7.5-5000$ & 1.7 & 0.1 & N/A & (29) \\
\hline Osmium polymer-SWCNT paste & Unsorted & 16 & $5-800$ & 5 & 0.2 & N/A & (16) \\
\hline Meldola’s blue/SWCNT/GC & Unsorted & 7.4 & $50-500$ & 0.048 & -0.1 & 6 & $(15)$ \\
\hline $\mathrm{PPF} /$ neutral red/SWCNT/PPF/Au & Unsorted & 29 & $80-4200$ & 18 & 0.15 & 7 & $(10)$ \\
\hline
\end{tabular}

(a) Evaluated by CV.

(b)MWCNT: multiwalled carbon nanotube.

(c)SPE: screen printed polymer.

The analytical performance was, at least, better than that with the unsorted SWCNT electrode in Table 1. Thus, the adoption of sorted SWCNTs is better in some cases or comparable to that for other sensors with the assistance of other functionalization elements such as electron transfer mediators and nanoparticles.

\subsubsection{Glucose sensing}

The GDH enzyme was used as a model biorecognition element to demonstrate a glucose biosensor based on the NADH sensor because NADH is a cofactor for a large number (more than 300) of NAD-dependent dehydrogenase enzymes. A preliminary investigation was performed to determine the effect of the SWCNT electronic type on the amperometric characteristics of a biosensor with NAD-dependent GDH..$^{(9)}$ The sensing mechanism involves a signal peak produced by NADH oxidation caused by the enzymatic reaction:

$$
\beta \text {-D-glucose }+\mathrm{NAD}^{+} \stackrel{\mathrm{GDH}}{\rightarrow} \delta \text {-gluconolactone }+\mathrm{NADH}
$$

The substrate (glucose) and coenzyme $\left(\mathrm{NAD}^{+}\right)$were simultaneously positioned in the vicinity of the active site of the enzyme (GDH). $\mathrm{NAD}^{+}$is a major electron acceptor in the oxidation of glucose, and the nicotinamide ring of $\mathrm{NAD}^{+}$accepts a hydrogen ion and two electrons, equivalent to a hydride ion. The reduced form of this carrier generated by the reaction is NADH, which can be electrochemically oxidized, i.e., electron transfer occurs at the anode via SWCNTs, as shown in Eq. (5). The fabrication process is continuous with NADH sensing. The GDH enzyme is added to obtain the glucose biosensor as a multilayer of PPF/GDH/PPF/SWCNT/PPF/Au. 
Figure 6 shows CV profiles for sensors with the unsorted SWCNT-GDH, m-SWCNT-GDH, and s-SWCNT-GDH electrodes. All electrodes showed an increase in current with glucose addition. The response of the s-SWCNT-GDH electrode was larger than those of the m-SWCNT-GDH and unsorted SWCNT-GDH electrodes. The current increase and CV profiles of the SWCNT-GDH electrode strongly correlated with those for the SWCNT electrodes (no enzyme) with NADH sensing shown in Fig. 5. However, the potential $(+0.6 \mathrm{~V})$ for an increase in current was larger than that for NADH oxidation with the SWCNT electrodes $(+0.4 \mathrm{~V})$. This is attributed to the overpotential due to the presence of the electronically insulating GDH layer. Figure 7(a) shows the

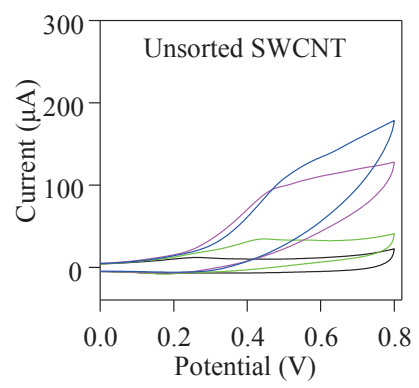

(a)

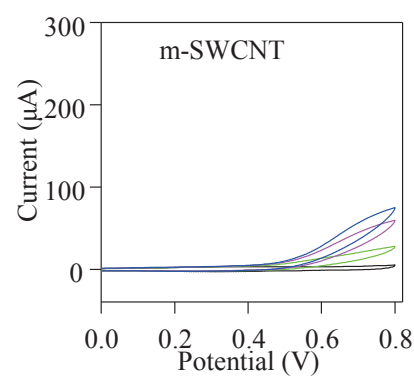

(b)

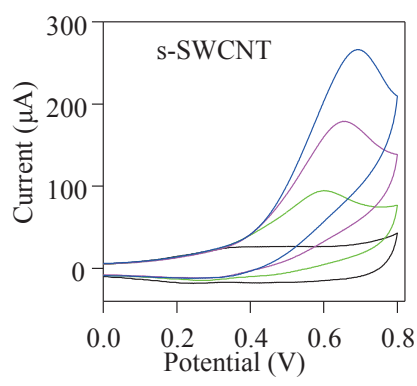

(c)

Fig. 6. (Color online) CV profiles for the GDH biosensors with (a) unsorted SWCNT, (b) m-SWCNT, and (c) s-SWCNT electrodes. Sweep rate: $50 \mathrm{mV} \mathrm{s}^{-1}$. Glucose concentration: 0, 2.5, 14, $48 \mathrm{mM}$. pH $7.420 \mathrm{mM}$ phosphate buffer solution.

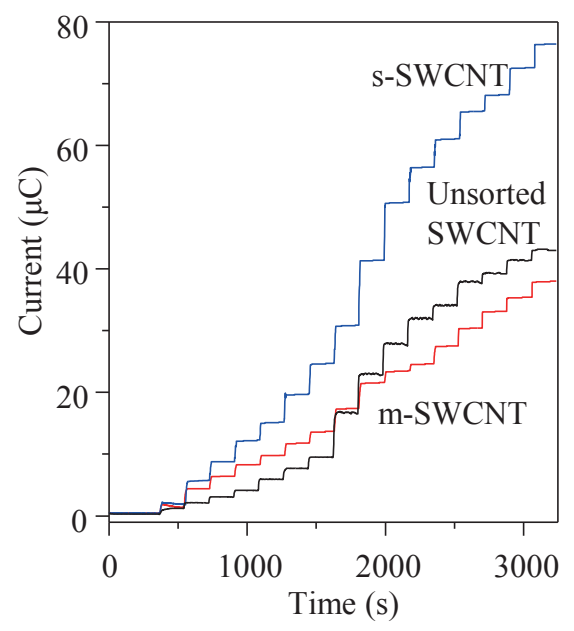

(a)

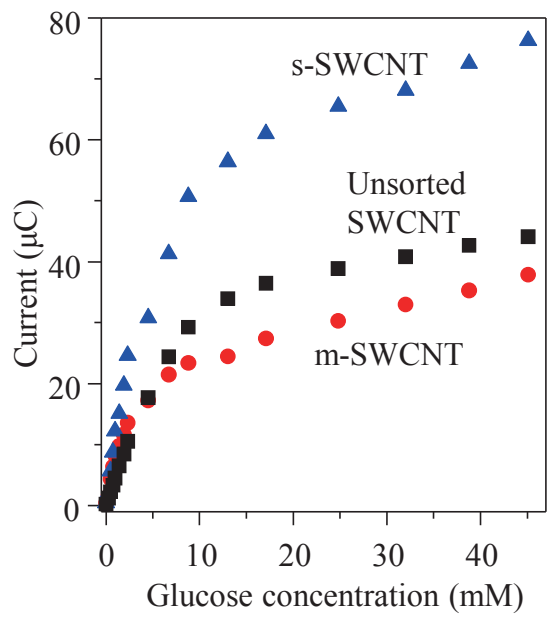

(b)

Fig. 7. (Color online) (a) Time-current response for sequential glucose addition at concentrations of $0,0.25$, $0.49,0.73,0.96,1.4,1.9,2.3,4.5,6.7,8.8,13,17,25,32,39$, and $45 \mathrm{mM}$ for unsorted-, m-, and s-SWCNT-GDH electrodes. The polarization potential was $+0.6 \mathrm{~V}$ vs $\mathrm{Ag} / \mathrm{AgCl}$ with an electrolyte of $\mathrm{pH} 7.420 \mathrm{mM}$ phosphate buffer solution. (b) Calibration plot for glucose response using the data in (a). The sensitivity of the s-SWCNTGDH electrodes was $45 \mathrm{~mA} \mathrm{mM}^{-1} \mathrm{~cm}^{-2}(r=0.991$ in the linear range of $0.25-2.5 \mathrm{mM})$. 


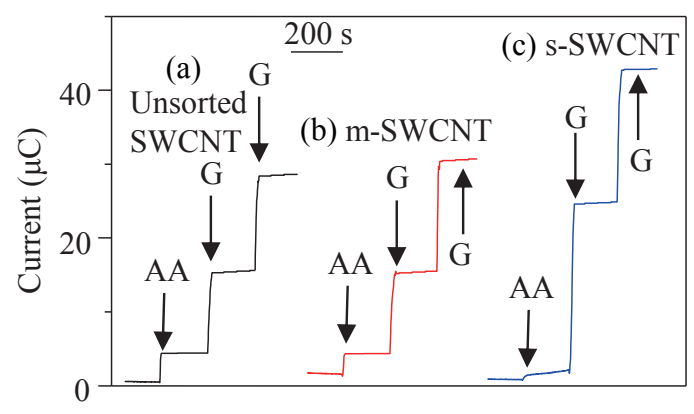

Fig. 8. (Color online) Amperometric response to $0.1 \mathrm{mM}$ ascorbic acid (AA) and 2.5, $4.9 \mathrm{mM}$ glucose (G) for (a) unsorted-, (b) m-SWCNT-, and (c) s-SWCNT-GDH electrodes. The polarized potential was $+0.6 \mathrm{~V}$ vs Ag/AgCl. The electrolyte was $\mathrm{pH}$ 7.4, $20 \mathrm{mM}$ phosphate buffer solution.

steady-state amperometric response at $+0.6 \mathrm{~V}$ for the SWCNT-GDH electrodes. A stepped increase in current was observed with glucose addition, the increase of which also correlated with the CV profile. Figure 7(b) shows the current vs glucose concentration based on the data from Fig. 7(a). The electrochemical responses were sufficiently differentiated to cover the clinically significant concentration range of glucose within the dynamic range of the sensor. Unlike NADH sensing, a deviation from linearity (saturation) was observed at higher $(>20 \mathrm{mM})$ glucose concentrations, which represents a typical characteristic of the Michaelis-Menten model for an enzymatic reaction. Figure 8 shows a comparison of the amperometric response in the presence of an interferant (ascorbic acid) and that due to glucose. The sSWCNT-GDH electrode showed the best selectivity for the target (glucose) compared to unsorted- and m-SWNCT electrodes. It has a strong correlation with the signal of glucose (Fig. 7). This is because the s-SWCNT-GDH electrode provides the best electrochemical configuration. All electrodes showed that the response by interferants was negligible, suggesting that this type of sensor is suitable for use in physiological samples.

\section{Conclusions}

The electrochemical behavior and analytical applications of electronically type-sorted SWCNT electrodes were demonstrated for the first time. The aim of this research is to obtain a fundamental understanding of the electrochemical properties of electronically type-sorted SWCNTs in a sensor electrode. Electronically type-sorted SWCNT electrodes were fabricated using a layer-by-layer process to combine PPFs as an inactive matrix. The electrodes exhibited contrasting responses to NADH. CV measurements showed that the current due to NADH oxidation with the s-SWCNT electrode was much larger than that with the m-SWCNT and unsorted SWCNT electrodes. EIS measurements showed that the s-SWCNT network was less resistant to electron transfer than the m-SWCNT network. Chronocoulometry measurements suggested that there was no distinct difference in the diffusion coefficient of NADH between the m-SWCNT and s-SWCNT electrodes. We conclude that s-SWCNTs are more suitable than m-SWCNTs and unsorted SWCNT for NADH detection with respect to catalysis and electron transfer. PPF modification of electronically typesorted SWCNTs is an effective strategy for an electrochemical communication-friendly platform 
in amperometric chemical sensors. The method presented here has the potential for application to various electrochemical sensors but could also be extended to biosensors and biofuel cells involving biological materials.

\section{Acknowledgements}

This work was supported by a Grant-in-Aid for Scientific Research (C) JSPS KAKENHI Grant Number 24560965.

\section{References}

1 F. Lu, M. J. Meziani, L. Cao, and Y. -P. Sun: Langmuir 27 (2010) 4339.

2 J. M. Schnorr and M. Swager: Chem. Mater. 23 (2011) 646.

3 M. S. Arnold, A. A. Green, J. F. Hulvat, S. I. Stupp, and M. C. Hersam: Nat. Nanotechnol. 1 (2006) 60.

4 A. L. Antaris, J. T. Seo, A .A. Green, and M. C. Hersam: ACS Nano 4 (2010) 4725.

5 M. Engel, J. P. Small, M. Steiner, M. Freitag, A. A. Green, M. C. Hersam, and P. Avouris: ACS Nano 2 (2008) 2445.

6 M. E. Roberts, M. C. LeMieux, and Z. Bao: ACS Nano 3 (2009) 3287.

7 M. Ganzhorn, A. Vijayaraghavan, S. Dehm, F. Hennrich, A. A. Green, M. Fichtner, A. Voigt, M. Rapp, H. von Löhneysen, M. C. Hersam, M. M. Kappes, and R. Krupke: ACS Nano 5 (2011) 1670.

8 A. P .F. Turner, I. Karube, and G. S. Wilson, Editors: Biosensors: Fundamentals and Applications, (Oxford University Press, Oxford, 1987).

9 H. Muguruma, T. Hoshino, and K. Nowaki: ACS Appl. Mater. Interfaces 7 (2015) 584.

10 T. Hoshino and H. Muguruma: IEICE Trans. Electron. E95-C (2012) 1300.

11 J. Filip, J. Ŝefčovičová, P. Tomčík, P. Gemeiner, and J. Tkac: Talanta 84 (2011) 355.

12 F. S. Saleh, T. Okajima, F. Kitamura, L. Mao, and T. Ohsaka: Electrochim. Acta 56 (2011) 4916.

13 A. Radoi, D. Compagnone, M. A. Valcarcel, P. Placidi, S. Materazzi, D. Moscone, and G. Palleschi: Electrochim. Acta 53 (2008) 2161.

14 P. Du, P. Wu, and C. Cai: J. Electroanal. Chem. 624 (2008) 21.

15 L. Zhu, J. Zhai, R. Yang, C. Tian, and L. Guo: Biosens. Bioelectron. 22 (2007) 2768.

16 R. Antiochia and L. Gorton: Biosens. Bioelectron. 22 (2007) 2611.

17 J. Liu, S. Tian, and W. Knoll: Langmuir 21 (2005) 5596

18 J. Wang and M. Musameh: Anal. Chem. 75 (2003) 2075.

19 H. Muguruma, Y. Shibayama, and Y. Matsui: Biosens. Bioelectron. 23 (2008) 827.

20 H. Muguruma, A. Hiratsuka, and I. Karube: Anal. Chem. 72 (2000) 2671.

21 H. Wang, G. Koleilat, P. Liu, G. Jiménez-Osés, Y. -C. Lai, M. Vosgueritchian, Y. Fang, S. Park, K. N. Houk, and Z. Bao: ACS Nano 8 (2014) 2609.

22 J. Chen, T. O. Tran, M. T. Ray, D. B. Brunski, J. C. Keay, D. Hickey, M. B. Johnson, D. T. Glatzhofer, and D. W. Schmidtke: Langmuir 29 (2013) 10586.

23 J. Moiroux and P. J. Elving: Anal. Chem. 50 (1978) 1056.

24 M. Wooten and W. Gorski: Anal. Chem. 82 (2010) 1299.

25 H. Teymourian, A. Salimi, and R. Hallaj: Biosens. Bioelectron. 33 (2012) 60.

26 J. Shalini, K. J. Sankaran, H. -C. Chen, C. -Y. Lee, N. -H. Taia, and I. -N. Lin: Analyst 139 (2014) 778.

27 A. J. Bard and L. R. Faulkner: Electrochemical methods: fundamentals and applications, (John Wiley \& Sons, New York, 1980).

28 R. S. Dey, S. Gupta, R. Paira, and C. R. Raj: ACS Appl. Mater. Interfaces 2 (2010) 1355.

29 A. Raoi, D. Compagnone, M. A. Valcarcel, P. Placidi, S. Materazzi, D. Moscone, and G. Pallcidi: Electrochim. Acta 53 (2008) 2161.

30 Y. Matsui, T. Hoshino, M. Yoshizawa, and H. Muguruma: Electrochemistry 76 (2008) 610.

31 A. Devadoss, J. W. Lee, C. Terashima, A. Fujishima, Y. -P. Kim, J. K. Kang, and U. Paik: Sens. Actuators, B 208 (2015) 204.

32 C. Deng, J. Chen, X. Chen, C. Xiao, Z. Nie, and S. Yao: Electrochem. Commun. 10 (2008) 907. 